VOL. 40 (1989) [235-241]

\title{
FREE FIBONACCI ALGEBRAS
}

\author{
D.L. Johnson AND A.C. KIM \\ Dedicated to Professor B.H. Neumann \\ on his eightieth birthday
}

\begin{abstract}
Fibonacci varieties were introduced by one of us in 1978 and a natural generalisation was studied shortly afterwards. We carry this investigation one stage further by giving a description of the free objects in these varieties. This is done in terms of the $n$-abelian groups of Levi.
\end{abstract}

\section{INTRODUCTION}

We are concerned with the variety $\mathcal{V}(m)$ of universal algebras $G$ of the following kind : $G$ is a group equipped with a unary operation $\phi$ that is an automorphism of $G$ and satisfies the one-variable law

$$
x x \phi \ldots x \phi^{m-1}=x \phi^{m}
$$

where $m$ is a positive integer and any occurrence of $\phi$ or one of its powers is understood to apply only to the symbol immediately preceding it. It seems natural to call such objects Fibonacci algebras, or $\phi$-algebras for short, in contradistinction to Fibonacci groups [2]. Note that we omit the condition in [4] that $\phi$ have some specified finite order, and hope to extend our results to this "modular" case in a future article. Our clief aim is to prove the following

THEOREM. The free object of rank $d$ in $\mathcal{V}(m)$ is given by

$$
V_{d}(m)=Z^{\times(m-1) d} \times F /\left(F^{\prime} \cap F^{m} \cap F^{m-1}\right)\left(F^{\prime} \cap F^{m-1} \cap F^{m-2}\right)
$$

where the first factor is the free abelian group of rank $(m-1) d$ and, in the second, $F=F_{d}$ is the (absolutely) free group of rank $d, F^{\prime}$ is its derived group, and $F^{m}$ the subgroup generated by all $m$ th powers.

\section{Received 30 March 1989}

Both authors are graleful to the British Council, the Royal Society of London, and the Korean Science and Engineering Foundation, without whose continued support this collaboration would not have been possible.

Copyright Clearance Centre, Inc. Serial-fee code: $0004-9729 / 89 \quad \$ A 2.00+0.00$. 


\section{Preliminaries}

We begin with a brief review of the main results of [4].

By evaluating the product $x x \phi \ldots x \phi^{m-1} x \phi^{m}$ in two different ways, we obtain the one-variable law

$$
x x \phi^{m+1}=x \phi^{m} x \phi^{m}
$$

Next, apply (1) to $x y$ :

$$
\begin{aligned}
x \phi^{m} y \phi^{m}=(x y) \phi^{m} & =x y(x y) \phi \ldots(x y) \phi^{m-1} \\
& =x y x \phi y \phi \ldots x \phi^{m-1} y \phi^{m-1} \\
& =x(y x \phi)(y x \phi) \phi \ldots(y x \phi) \phi^{m-1} x^{-1} \phi^{m} \\
& =x(y x \phi) \phi^{m} x^{-1} \phi^{m}, \text { applying (1) to } y x \phi \\
& =x y \phi^{m} x \phi^{m+1} x^{-1} \phi^{m} \\
& =x y \phi^{m} x^{-1} x \phi^{m}, \text { by }(2) .
\end{aligned}
$$

Thus, $x^{-1} x \phi^{m}$ commutes with $y \phi^{m}$ and so is central. But

$$
x^{-1} x \phi^{m}=x \phi^{m+1} x^{-1} \phi^{m}=\left(x \phi x^{-1}\right) \phi^{m}
$$

by (2), and we have the following central result.

Proposition 1. For all $x \in G, x^{-1} x \phi$ is central.

It follows easily that the set

$$
H=\left\{x^{-1} x \phi \mid x \in G\right\}
$$

is closed under multiplication, inverses, and the action of $\phi$, so that $G / H \in \mathcal{V}(m)$ and admits the trivial $\phi$-action. It is thus the largest $\phi$-trivial factor-algebra $G^{\phi}$ of $G$. If we let $G_{\phi}$ denote the largest $\phi$-trivial subalgebra of $G$, then Proposition 1 can be restated as follows.

Corollary 1. The mapping $\theta: G \rightarrow G, x \mapsto x^{-1} x \phi$, is a homomorphism into $Z(G)$ with $\operatorname{Ker} \theta=G_{\phi}$ and $\operatorname{Coker} \theta=G^{\phi}$.

Since $\phi$-trivial groups have exponent dividing $m-1$, it follows that $G^{m-1} \leqslant \operatorname{Im} \theta \leqslant$ $Z(G)$, and we have

Corollary 2. The law $\left[x^{m-1}, y\right]=1$ holds in $G$.

This is (1.4) in Theorem 3 of [4], and it follows that the algcbras in $\mathcal{V}(2)$ are all commutalive, which is the Lemma in [3].

By applying Proposition 1 to $x, x \phi, x \phi^{2}, \ldots$ in turn, it follows that $x^{-1} x \phi^{k}$ is central for all $k \in \mathrm{N}$, whence the images of $x$ under all integral powers of $\phi$ commute in pairs, which yields Theorem 1 of [4]: 
Corollary 3. The monogenic free algebra in $\mathcal{V}(m)$ is commutative.

Next, apply (1) to $x y$ as above to obtain:

$$
x x \phi \ldots x \phi^{m-1} y y \phi \ldots y \phi^{m-1}=x y x \phi y \phi \ldots x \phi^{m-1} y \phi^{m-1} .
$$

Since the terms $x, x \phi, \ldots, x \phi^{m-1}$ each appear once on both sides and are congruent to $x$ modulo $Z(G)$, they can all be replaced by $x$. Similarly, each of $y, y \phi, \ldots, y \phi^{m-1}$ can be replaced by $y$.

Corollary 4. The law $x^{m} y^{m}=(x y)^{m}$ holds in $G$.

This law defines the variety $\mathcal{A}(m)$ of $m$-abelian groups introduced in $[5]$ and classified in [1]. In view of the fact that any two of the laws

$$
\left[x^{m-1}, y\right]=1, \quad x^{m} y^{m}=(x y)^{m}, \quad x^{m-1} y^{m-1}=(x y)^{m-1}
$$

imply the third (easy exercise), Corollaries 2 and 4 can be combined into

Corollary 5. $\mathcal{V}(m) \leqslant \mathcal{A}(m) \cap \mathcal{A}(m-1)$.

It follows that the algebras of $\mathcal{V}(3)$ are commutative, which is Theorem 2 of [4].

\section{A CRITERION}

For every $x$ in a $\phi$-algebra $G$, the $\phi$-subalgebra generated by $x$ is abelian (Corollary 1.3); we write it additively for the moment and work with the homomorphisms

$$
\theta: x \mapsto-x+x \phi, \quad \mu:(m-1) x
$$

noting that $\operatorname{Im} \mu \leqslant \operatorname{Im} \theta \leqslant Z(G)$. For each $x \in G$ and every $k=0,1,2, \ldots$, we have

$$
x \phi^{k}=x(1+\theta)^{k}
$$

and (1) can be rewritten in the form

$$
m x+\sum_{k=0}^{m-1} x\left\{(1+\theta)^{k}-1\right\}=x+x\left\{(1+\theta)^{m}-1\right\}
$$

that is,

$$
x \mu=x f_{m}(\theta), \quad \text { where } f_{m}(t) \in \mathbf{Z}[t]
$$

is a monic polynomial of degree $m$ with zero constant term. Since $\theta$ is a homomorphisin with $\operatorname{Im} \theta \subseteq Z(G)$, the same is true of $f_{m}(\theta)$, and we have another proof of Corullary 1.2. We also have the following characterisation of $\phi$-algebras. 
Proposition 2. Let $G$ be any group, $X$ a set of generators for $G$, and $\phi$ an automorphism of $G$ such that :

(i) (1) holds for all $x \in X$,

(ii) $x^{-1} x \phi \in Z(G), \forall x \in X$,

(iii) the map $\mu: g \mapsto g^{m-1}$ is a homomorphism.

Then (1) holds for all $x \in G$, that is, $G$ is a $\phi$-algebra.

Proof: Since $\phi$ is a homomorphism, it follows from (ii) that $g^{-1} g \phi \in Z(G)$ for all $g \in G$, and that $\theta: g \mapsto g^{-1} g \phi$ is a homomorphism. Thus, using (i), (5) holds for all $x \in X$, and so for all $x \in G$, by (iii). Since every $x \in G$ commutes with all the $x \phi^{k}, k \in N,(1)$ now follows from (5).

Corollary 1. If $G$ is an abelian group and $\phi \in$ Aut $G$ satisfies (1) on a set of generators of $G$, then (1) is a law in $G$.

Corollary 2. Let $G$ be a group and $\theta: G \rightarrow Z(G)$ a homomorphism such that

$$
(m-1) g=g f_{m}(\theta), \quad \forall g \in G,
$$

where $f_{m}(t) \in Z[t]$ is given by

$$
f_{m}(t)=(1+t)^{m}-\frac{(1+t)^{m}-1}{t}+(m-1) .
$$

Then $G$ is a $\phi$-algebra with respect to $\phi: g \mapsto g(1+\theta)$.

\section{FREe objects in $\mathcal{V}(m)_{a b}$}

Because of Corollary 2.2 , the direct product of two $\phi$-algebras is a $\phi$-algebra in the natural way. Moreover, when they are abelian, it is their free product in $\mathcal{V}(m)_{a b}$. Since the free object of rank $d$ in any variety is just the $d$ th free power of the monogenic free object, it suffices to describe the latter. Consider the group

$$
F(m)=\left\langle x \mid Y_{m}, C\right\rangle
$$

where

$$
X=\left\{x_{i} \mid i \in \mathbf{Z}\right\}
$$

$$
\begin{gathered}
Y_{m}=\left\{x_{i} x_{i+1} \ldots x_{i+m-1} x_{i+m}^{-1} \mid i \in \mathbf{Z}\right\}, \\
C=\left\{\left[x_{i}, x_{j}\right] \mid i, j \in \mathbf{Z}\right\},
\end{gathered}
$$

which is clearly free abelian of rank $m$. Now the map $\phi: x_{i} \mapsto x_{i+1}$ clearly extends to an automorphism of $F(m)$ satisfying (1) for all $x \in X . F(m)$ is thus a $\phi$-algebra by Corollary 2.1, and generaled as such by $x_{0}$. Given any element $y$ in any $G$ in $\mathcal{V}(m)_{a b}$, it is clear how to define a $\phi$-homomorphism from $F(m)$ to $G$ sending $x_{0}$ to $y$. Thus $F(m)$ is the monogenic free object in $\mathcal{V}(m)_{a b}$. 
Proposition 3. The free object of rank $d$ in $\mathcal{V}(m)_{a b}$ is the $\phi$-algebra $F(m)^{x d}$, where $F(m)$ is defined by (6) and (7).

Corollary 1. $F(m)$ is the monogenic free object in $\mathcal{V}(m)$.

Corollary 2. $F(m)^{\times d}$ is the free object of rank $d$ in $\mathcal{V}(m)$ when $m=2$ or 3 .

These are consequences of Corollary 1.3 and the fact that $\phi$-algebras are abelian when $m=2$ or 3 , respectively.

For later use, we replace the basis $\left\{x_{i} \mid 0 \leqslant i \leqslant m-1\right\}$ of $F(m)$ by

$$
x=x_{0}, \quad y_{i}=x_{i-1}^{-1} x_{i}, \quad 1 \leqslant i \leqslant m-1
$$

then $F(m) \theta$ has basis $x^{m-1}, y_{i}, 1 \leqslant i \leqslant m-1$.

\section{FreE products in $\mathcal{V}(m)$}

Given $\phi$-algebras $G$ and $I I$, out strategy is to factor out from their ordinary free product $F:=G * H$ "just enough" extra relators to yield a $\phi$-algebra $G *_{\phi} H$. Specifically, let $G$ and $H$ be presented as groups by

$$
G=\langle X \mid R\rangle, \quad H=\langle Y \mid S\rangle
$$

so that

$$
F=\langle X, Y \mid R, S\rangle
$$

Now $\phi$ is defined on the generators $X \cup Y$; let $\nu: F \rightarrow K$ be any group homomorphism into a $\phi$-algebra $K$ that commutes with $\phi$ on $X \cup Y$. Then $\nu$ annihilates all $\left[z \theta, z^{\prime}\right]$, $z, z^{\prime} \in X \cup Y, \theta$ as above. Moreover, if $w=w(X \cup Y)$ is any word in $\langle X, Y \mid\rangle$, then $w \mu=w^{m-1}$ and $w((X \cup Y) \mu)$ have the same image under $\nu$. (The latter word is that obtained from $w$ by replacing each letter by its $(m-1)$ th power.) Now hopefully put

$$
G *_{\phi} H=\langle X, Y \mid R, S,[X \theta, Y],[X, Y \theta], W\rangle,
$$

where $\theta$ is defined on $X$ and $Y$ as in (4), and

$$
W=\left\{w^{m-1} w((X \cup Y) \mu)^{-1} \mid w \in(X, Y|\rangle\}\right.
$$

From what has been said, any $\nu$ of the above type factors through $G *_{\phi} H$. Furthernore, the group presented in (9) clearly satisfies all the conditions of Proposition 2 . It is thus the biggest $\phi$-homomorplic image of $F$, and as such is the free product of $G$ and $H$ in $\mathcal{V}(m)$. 
Proposition 4. The free product of groups $G, H$ in $\mathcal{V}(m)$ is given by (9).

Corollary 1. The free object of rank $d$ in $\mathcal{V}(m)$ is $F(m)^{* \phi^{d}}$.

Now consider the result $\left(G *_{\phi} H\right)^{\phi}$ of factoring $G *_{\phi} H$ out by its central subgroup $\langle X \theta, Y \theta\rangle$. The relators $[X \theta, Y],[X, Y \theta]$ become redundant and $W$ reduces to

$$
P=\left\{w^{m-1} \mid w \in\langle X, Y \mid\rangle\right\}
$$

as $X_{\mu} \subseteq G \theta, Y \mu \subseteq H \theta$. It follows that $\left(G *_{\phi} H\right)^{\phi}$ has the presentation

$$
\langle X, Y \mid R, S, X \theta, Y \theta, P\rangle=G^{\phi} *_{B} H^{\phi}
$$

where the right-hand side is the free product in the Burnside variety $\mathcal{B}(m-1)$ of groups of exponent $m-1$. Because of the natural $\phi$-homomorphism from $G{ }^{*} \phi H$ onto $G \times H$, it is clear that

$$
\langle X \theta, Y \theta\rangle=\left(G *_{\phi} I I\right) \theta=G \theta \times H \theta .
$$

Corollary 2. There is a central extension

$$
1 \rightarrow G \theta \times H \theta \rightarrow G *_{\phi} H \rightarrow G^{\phi} *_{B} H^{\phi} \rightarrow 1
$$

COROLlaky 3. $G *_{\phi} H$ is the result of factoring out $G * H$ by the intersection of the kernels of the natural maps onto $G, H$ and $G^{\phi} *_{B} H^{\phi}$.

Conollany 4. The free object $V_{d}(m)$ of rank $d$ in $\mathcal{V}(m)$ is a central extension of the corresponding object in $\mathcal{B}(m-1)$ by a free abelian group of rank $m d$.

Corollary 3 follows at once from Corollary 2, and Corollary 4 by induction on $d$ from Corollaries 1 and 2 .

\section{FREE OBJECTS IN $\mathcal{V}(m)$}

We conclude by describing the presentation of $V_{d}(m)=F(m)^{*_{\phi}{ }^{d}}$ arrived at using (9), where each $\phi$-free factor is generated by a set of the form (8). We refer to these $m$ generators of the $i$ th factor as $i$-generators, $1 \leqslant i \leqslant d$, and to the $d$ generators $x$ and $(m-1) d$ generators $y_{i}, 1 \leqslant i \leqslant m-1$, as $x$-generators and $y$-generators respectively. Letting $Z$ denote the set of all such generators, the defining relators for $F(m)^{* \phi^{d}}$ are now of three types (corresponding respectively to $R$ and $S,[X \theta, Y]$ and $[X, Y \theta], W$ respectively).

(1) the $m$ i-generators commute along themselves, $1 \leqslant i \leqslant d$.

(2) the $y$-generators are all central, and so are the $(m-1)$ th powers of the $x$-generators.

(3) $w^{m-1}=w(Z \mu)$, for all $w \in\langle Z \mid\rangle$. 
Now the centrality of the $y$-generators asserted in (2) ensures that:

(i) the relators (1) are superfluous, and

(ii) only words $w$ in the $x$-generators are needed in (3).

It follows that $V_{d}(m)$ is the direct product of the subgroups $C$ and $A$ generated by the $x$-generators and $y$-generators, respectively. Moreover, it follows from Corollary 4.2 that $A$ is free abelian of rank $(m-1) d$, and that $C$ is the free object $C_{d}(m-1)$ of rank $d$ in the variety $\mathcal{C}(m-1)=\mathcal{A}(m) \cap \mathcal{A}(m-1)$ defined by the laws (3).

Proposition 5. $V_{d}(m) \cong C_{d}(m-1) \times Z^{\times(m-1) d}$.

The theorem is an immediate consequence of this, by a result of Alperin [1] which asserts that the free object of rank $d$ in $\mathcal{A}(m)$ is given by

$$
A_{d}(m)=\frac{F}{F^{\prime} \cap F^{m} \cap F^{m-1}},
$$

where $F=F_{d}$ is the (absolutely) free group of rank $d$.

Examples. Since $F^{0}=\{1\}, F^{1}=F$, and $F^{2} \supseteq F^{\prime}$, we read off

$$
C_{d}(0)=F, \quad C_{d}(1)=C_{d}(2)=F / F^{\prime}, \quad C_{d}(3)=F / F^{\prime} \cap F^{3} .
$$

Putting $d=2$ in the last case, it can be shown using Corollary 4.4 that

$$
\begin{aligned}
& F / F^{\prime} \cap F^{3} \\
& =\left\langle x, y \mid\left[x^{3}, y\right]=\left[x, y^{3}\right]=1,(x y)^{3}=x^{3} y^{3},\left(x y^{-1}\right)^{3}=x^{3} y^{-3}\right\rangle . \\
& \text { REFERENCES }
\end{aligned}
$$

\section{REFERENCES}

[1] J. L. Alperin, 'A classification of n-abelian groups', Canad. J. Math. 21 (1969), 1238-1244.

[2] D. L. Juhnson, J. W. Wamsley and D. Wright, 'The Fibonacci groups', Proc. London Math. Soc. (3) 10 (1974), 577-594.

[3] Ann Chi Kin, 'Fibonacci varieties', Bull. Austral. Math. Soc. 10 (1978), 191-196.

[4] Aun Chi Kim, B. II. Neumann and A. II. Rhemtulla, 'Mure Fibonacci varieties', Bull. Austral. Math. Soc. 22 (1980), 385-395.

[5] F. Levi, 'Notes on group theory', J. Indian Math. Soc. 8 (1944), 1-7.

Mathematics Department University of Nottingham Nottingham NG7 2RD England
Mathematics Department

Pusan National University

Pusan

Korea 607 